\title{
On Distribution of Aggregate Interference in Cognitive Radio Networks
}

\author{
Yaobin $\mathrm{Wen}^{\dagger}$, Sergey Loyka ${ }^{\dagger}$ and Abbas Yongacoglu ${ }^{\dagger}$
}

\begin{abstract}
This paper analyzes the distribution of aggregate interference in cognitive radio networks. Poisson point spatial distribution model and average propagation path loss model are considered. All possible scenarios are classified into three typical cases, based on typical outage events. When the average number of nodes in the forbidden region is much smaller than one, the aggregate interference can be well approximated by the nearest one (nearest node dominates outage events). When the average number of nodes in the forbidden range is greater than one, the aggregate interference can be approximated by a Gaussian random variable (many nodes contribute to outage). When the average number of nodes in the forbidden range is slightly smaller than one, neither the nearest node approximation nor Gaussian one is accurate (a few near-by nodes are dominant), and higher order cumulants approximations or others are required. We derive the nearest interference distribution and give a simpler way to calculate the cumulants of the aggregate interference.
\end{abstract}

\section{INTRODUCTION}

As higher data rate services are required in wireless communications, there is a need for more spectrum efficiency. To overcome the overcrowded spectrum problem and use spectrum more efficiently, Cognitive Radio (CR) suggests allowing the secondary users (SU) to share the spectrum which is not currently used by the primary user (PU). Spectrum sensing is used by SUs to find spectrum "holes" [1]. Due to the uncertainty of SU node's number and locations, PU performance may be seriously affected by the aggregate interference generated by SUs, so its accurate modeling is important to design cognitive radio networks.

To model the aggregate interference in a wireless network, we have to properly choose node spatial distribution and propagation path loss models. The most popular spatial distribution model is a Poisson point process on a plane. Based on this model and average propagation path loss model, Sousa and Silvester [2] studied the aggregate interference power. They obtained its characteristic function $(\mathrm{CF})$ and concluded that the aggregate interference power is an $\alpha$ - stable random variable. In some special cases, the closed-form probability density function (PDF) was derived from its CF. Sousa [3] extended that model and studied the aggregate interference as a random vector at the output of receiver correlators. He obtained the joint $\mathrm{CF}$ of the aggregate interference and concluded that the aggregate interference is a symmetric $\alpha$ - stable random vector. By using the multivariate Lepage series representation, Ilow and Hatzinakos [4] obtained the CF

$\dagger$ Y. Wen, S. Loyka and A. Yongacoglu are with the School of Information Technology and Engineering, University of Ottawa, Ontario, Canada, K1N 6N5, e-mails: ywen027@uottawa.ca, sergey.loyka@ieee.org, yongacog@site.uottawa.ca. of the aggregate interference in the plane/volume according to a Poisson point process. They also concluded that the aggregate interference is a spherically symmetric $\alpha$ - stable random vector. In their model, lognormal and Rayleigh fading were taken into account. Mordachev and Loyka [5] studied the relationship of the outage probability and the node density in wireless networks. Poisson point process, the average propagation path loss, different fading models and interference cancellation were considered. By studying the tail of the aggregate interference power distribution, they found that, at the low outage region, the aggregate interference is dominated by the nearest one. Based on this theorem, a compact and closed-form outage probability of the aggregate interference was derived. Ghasemi and Sousa [6] studied the aggregate interference in cognitive networks. Poisson point process, the average propagation path loss and different fading models were considered. They used Campell's theorem to get the $\mathrm{CF}$ of the aggregate interference power. Based on the $\mathrm{CF}$, the cumulants of the aggregate interference were obtained. Since the closed-form PDF expression can not be found from the $\mathrm{CF}$ for most cases, they used those cumulants to generate an approximated distribution of the aggregate interference. They also studied the effect of cooperative sensing on the distribution of the aggregate interference under i.i.d. fading channels.

In cognitive radio networks, based on a typical CR protocol, SUs inside the forbidden range circle centered on the PU are not allowed to transmit, so the distribution of the aggregate interference is not an $\alpha$-stable distribution any more. The models of [2]-[5] are not suitable for cognitive radio networks. On the other hand, the approximated distribution of the aggregate interference in [6] only uses first three cumulants, and the accuracy of this approximation is poor when forbidden range is small and the interference node density is low.

To overcome these limitations, we develop a new method to study the distribution of aggregate interference in cognitive radio networks, based on Poisson point process and the average propagation path loss models. All possible scenarios are classified into three typical cases, based on typical (dominant) outage events (i.e. when the aggregate interference at the PU receiver exceeds a threshold):

- Case 1: when the average number of nodes in the forbidden range is much smaller than one, a typical dominant outage event is when the nearest node interference exceeds the threshold, and the aggregate interference can be well approximated by the nearest one. We give a PDF of the nearest interference and closed-form outage probability expression in terms of the interference to noise ratio (INR). 
- Case 2: when the average number of nodes in the forbidden range is greater than one, a typical outage event is when aggregate interference from many nodes exceeds the threshold. The aggregate interference can be approximated by a Gaussian random variable. We derive a simpler way to find the cumulants of the aggregate interference.

- Case 3: when the average number of nodes in the forbidden range is slightly smaller than one, a typical outage event is when the combination of a few nearest nodes interference exceeds the threshold. Neither the nearest node approximation nor Gaussian one is accurate in this case. Higher order cumulants approximations or others are required.

The paper is organized as follows. In Section II, the node spatial distribution model, CR protocol and propagation path loss model are introduced. Section III analyzes the distribution of the aggregate interference and outage probability in terms of the INR. The simulation results validate the analysis and approximations.

\section{SYSTEM MODEL}

We consider a cognitive radio network which contains a primary user (PU) receiver and many secondary user (SU) transmitters on a plane. The PU is located at the origin. The SUs are randomly located according to a Poisson point process. The density of SUs is $\lambda\left[\right.$ nodes $\left./ \mathrm{m}^{2}\right]$. Interference from the nodes outside the circle of a certain radius $R_{\max }$ is assumed to be negligible (alternatively, no SUs are located outside of this circle). The CR protocol is that all SUs which are inside of a forbidden region, i.e. the circle of the radius $R_{s}$ centered on the PU, cease their transmissions.

We assume the desired signal, interferences and noise are independent of each other. The received power of the PU can be expressed as:

$$
P_{y}=P_{x}+\sum_{i=1}^{N} I_{i}+P_{0}
$$

where $P_{x}$ is the desired signal power; $I_{i}$ is the interference signal power coming from the $i^{\text {th }} \mathrm{SU}$ node; $P_{0}$ is the noise power; $N$ is a Poisson random variable which denotes the number of nodes in the ring between circles of the radii $R_{s}$ and $R_{\max }$, i.e. the potential interference zone. We follow the propagation model which was used in [5]. The power at the receiver antenna output coming from the transmitter is $P_{r}=$ $P_{t} G_{t} G_{r} g$, where $P_{t}$ is the transmitter power; $G_{t}$ and $G_{r}$ are the transmitter and receiver antenna gain; $g$ is the propagation path loss. In this paper, only the average propagation path loss is considered, $g=a_{\nu} r^{-\nu}$, where $\nu$ is the path loss exponent, $r$ is the distance between the transmitter and receiver, and $a_{\nu}$ is a constant independent of $r$. For simplicity, we assume the transmitter and receiver antennas are isotropic, so that $P_{r}=$ $P_{t} a_{\nu} r^{-\nu}$, and that all SUs transmit at the same constant power level $P_{s}$. The $i^{t h} \mathrm{SU}$ generates the interference power $I_{i}=$ $P_{s} a_{\nu} r_{i}^{-\nu}$ at the PU receiver, where $r_{i}$ is the distance between the $i^{t h} \mathrm{SU}$ and PU. Without loss of generality, we normalize $P_{s} a_{\nu}=1$, so $I_{i}=r_{i}^{-\nu}$. Let $R_{0}=P_{0}^{-1 / \nu}$, so the interference level is below the noise level outside the circle of the radius $R_{0}$.

\section{OUTAGE PROBABILITY ANALYSIS AND SIMULATION} RESULTS

When signal to interference plus noise ratio (SINR) is less than a threshold $\eta$, there is significant performance degradation of a wireless link and the receiver is considered to be in outage. The probability of SINR being less than $\eta$ is an outage probability. If the signal and noise power are fixed, the outage probability is the probability of aggregate interference $I_{a g}$ exceeding the threshold $I_{t h}=P_{x} / \eta-P_{0}$,

$$
P_{\text {out }}=\operatorname{Pr}\{\operatorname{SINR}<\eta\}=\operatorname{Pr}\left\{I_{a g}>I_{\text {th }}\right\}
$$

Defining the interference to noise ratio (INR) as

$$
\gamma=\frac{\sum_{i=1}^{N} I_{i}}{P_{0}}
$$

its threshold value is $D=I_{t h} / P_{0}$, so that the outage probability is:

$$
P_{\text {out }}=\operatorname{Pr}\{\gamma>D\}=1-F(D)
$$

where $F(D)$ is the CDF of INR.

Let $\operatorname{Disk}(r)$ be a disk of the radius $r$, and $\operatorname{Ring}\left(r_{1}, r_{2}\right)$ be a ring between the circles of the radii $r_{1}$ and $r_{2}$, where $r_{2} \geq r_{1}$. The interference from a single $\mathrm{SU}$ in the disk of the radius $R(D)=\left(D P_{0}\right)^{-1 / \nu}$ results in the INR greater than $D$.

When all SUs are allowed to transmit, from [5] Theorem 1,

$$
\lim _{x \rightarrow \infty} \frac{\operatorname{Pr}\left\{\sum_{i=1}^{N} I_{i}>x\right\}}{\operatorname{Pr}\left\{I_{1}>x\right\}}=1, I_{1} \geq I_{2} \geq \cdots \geq I_{N}
$$

where $I_{1}$ is the strongest interference. Considering our propagation model, the strongest interference is coming from the nearest SU. Based on (5), we know that the aggregate interference is dominated by the nearest interference at the low outage region, so that from [5],

$$
P_{\text {out }} \approx \begin{cases}1, & D \leq D_{0} \\ \bar{N}_{0} D^{\frac{-2}{\nu}}, & D>D_{0}\end{cases}
$$

where $\bar{N}_{0}=\pi \lambda R_{0}^{2}$ is the average number of nodes in $\operatorname{Disk}\left(R_{0}\right) ; D_{0}=\bar{N}_{0}^{\nu / 2}$ is a critical value which separates the high and low outage probability regions. It corresponds to on average one SU being in the disk of the radius $R\left(D_{0}\right)=$ $\left(D_{0} P_{0}\right)^{-1 / \nu}=(\pi \lambda)^{-1 / 2}$, so that the outage probability is high if $D \leq D_{0}\left(\right.$ since $\left.R(D) \geq R\left(D_{0}\right)\right)$.

When the CR protocol is implemented, all SUs inside the forbidden range $R_{s}$ cease their transmissions. The interference generated by a single node can not exceed $I_{\max }=R_{s}^{-\nu}$, i.e. the value coming from an active node at the closest possible distance, so that the maximum INR from a single node is $D_{\max }=I_{\max } / P_{0}$.

Based on relationship between $R\left(D_{0}\right)$ and $R_{s}$, we classify all possible scenarios into three different cases and derive corresponding approximations for the outage probability. 


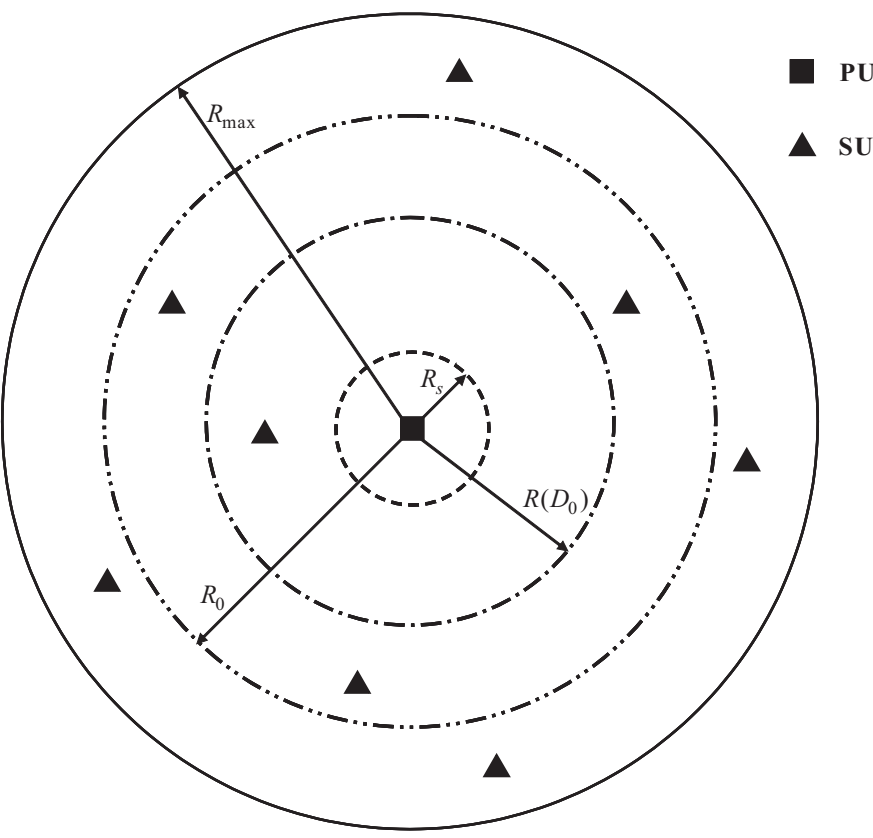

Fig. 1. The geometry of Case 1. Interference from the nodes outside the circle of the radius $R_{\max }$, is small and not considered. Outside the circle of the radius $R_{0}$, the interference level is below the noise level. The average number of nodes in the disk of the radius $R\left(D_{0}\right)$ is one. $R_{s}$ is the forbidden range, and all SUs inside the circle of the radius $R_{s}$ centered on the PU cease their transmissions.

A. Case 1: $R_{s} \ll R\left(D_{0}\right)$, and the aggregate interference is approximated by the nearest one for $D<D_{\max }$.

The geometry of Case 1 is illustrated by the Fig. 1.

Let $\operatorname{Ring}\left(R_{s}, R(D)\right)$ be a first ring, and $\operatorname{Ring}(R(D /(k-$ $1)), R(D / k)), k=2,3, \ldots, n, \ldots$ be the $k^{t h}$ ring. Since $k^{2 / \nu}-(k-1)^{2 / \nu}$ is a decreasing function of $k$ when $\nu>2$, the area of the $k^{t h}$ ring is $\left[k^{2 / \nu}-(k-1)^{2 / \nu}\right] \pi R^{2}(D)$ decreases with $k$.

When $R_{s} \ll R(D), \pi R^{2}(D)$ is much greater than $\pi R_{s}^{2}$, so the area of the first ring is $\pi\left[R^{2}(D)-R_{s}^{2}\right] \approx \pi R^{2}(D)$. Since the nodes are located as a Poisson point process, the probability of $n$ nodes in the first ring is almost the same as that in $\operatorname{Disk}(R(D))$, so that, as far as the outage probability is concerned, $R_{s} \approx 0$. Therefore, we can use the corresponding results in [5], and the aggregate interference can be approximated by the nearest one, $\sum_{i=1}^{N} I_{i} \approx I_{1}$, when evaluating $P_{\text {out }}$.

When $R_{s}<R(D)$ and $R(D)$ is close to $R_{s}$, we have $R(D) \ll R\left(D_{0}\right)$ since $R_{s} \ll R\left(D_{0}\right)$. The probability of one or more nodes being in the first ring $\operatorname{Ring}\left(R_{s}, R(D)\right)$ is $P_{1}=1-\exp \left(-\lambda A_{1}\right) \approx \lambda A_{1}$, where $A_{1}=\pi\left[R^{2}(D)-R_{s}^{2}\right]$ is the area of the first ring, and the probability of no node being in the first ring and two or more nodes being in the second one, $\operatorname{Ring}(R(D), R(D / 2))$, is $P_{2}=\exp \left(-\lambda A_{1}\right)[1-$ $\left.\exp \left(-\lambda A_{2}\right)-\lambda A_{2} \exp \left(-\lambda A_{2}\right)\right] \approx\left(\lambda A_{2}\right)^{2} / 2$, where $A_{2}=$ $\pi\left[R^{2}(D / 2)-R^{2}(D)\right]$ is the area of the second ring. Since $R(D) \ll R\left(D_{0}\right)$, these two events are the dominant outage events (the probabilities of the outage events which involve nodes in other rings are quite small, such as the event that no node in the first ring, one node in the second ring and two nodes in third and fourth rings). Comparing $P_{1}$ and
$P_{2}$, when $R(D)>\sqrt{1+\lambda \pi R_{s}^{2}\left(2^{2 / \nu}-1\right)^{2} / 2} R_{s}, P_{1}>P_{2}$. Since $\lambda \pi R_{s}^{2}\left(2^{2 / \nu}-1\right)^{2} / 2 \ll 1\left(\lambda \pi R_{s}^{2} \ll \lambda \pi R^{2}\left(D_{0}\right)=1\right)$, $\sqrt{1+\lambda \pi R_{s}^{2}\left(2^{2 / \nu}-1\right)^{2} / 2} R_{s} \approx R_{s}$, so that if $R_{s}<R(D)$ and $R(D)$ is close to $R_{s}$, then the aggregate interference can still be approximated by the nearest one.

When $R_{s}>R(D)$, let $n=$ floor $\left(D / D_{\max }\right)$, where floor (.) is the floor function. The typical outage event is $n+2$ or more nodes being in $\operatorname{Ring}\left(R_{s}, R(D /(n+2))\right.$. The aggregate interference is dominated by a few nearest nodes, not only by the nearest one, so that the aggregate interference can not be accurately approximated by the nearest one in this region. However, since the outage probability is very small and drops very fast in this region, $P_{\text {out }} \approx 0$.

Based on Poisson point spatial distribution model, it is straightforward to get the CDF of the nearest interference:

$$
F_{1}(I)= \begin{cases}1 & I>I_{\max } \\ \exp \left[\lambda \pi\left(I_{\max }^{-2 / \nu}-I^{-2 / \nu}\right)\right] & I \leq I_{\max }\end{cases}
$$

The outage probability in terms of the INR is

$$
P_{\text {out }} \approx \begin{cases}1 & D<D_{0} \\ 1-\exp \left[\bar{N}_{0}\left(D_{\max }^{\frac{-2}{\nu}}-D^{\frac{-2}{\nu}}\right)\right] & D_{0} \leq D<D_{\max } \\ 0 & D \geq D_{\max }\end{cases}
$$

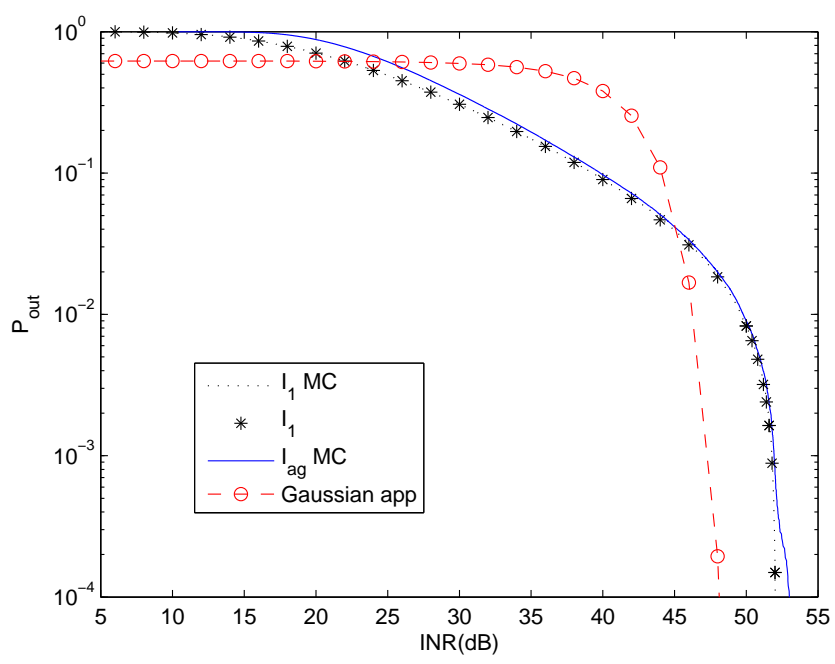

Fig. 2. Outage probability for Case $1 . \nu=4, R_{s}=10 \mathrm{~m}, R\left(D_{0}\right)=56.4 \mathrm{~m}$, $R_{0}=200 \mathrm{~m}, R_{\max }=10^{3} \mathrm{~m}$ and $\lambda=1 \times 10^{-4}\left[\right.$ nodes $\left./ \mathrm{m}^{2}\right]$. The dot line denotes the simulation result of the nearest interference; the star denotes theoretical result of the nearest interference; the solid line denotes the simulation result of the aggregate interference; the dash line with circles denotes Gaussian approximation. Based on the parameter above, $D_{0}=22 \mathrm{~dB}, D_{\max }=52 \mathrm{~dB}$.

Fig. 2 shows the simulation result for Case 1. From Fig. 2, when INR threshold is less than $D_{0}, P_{\text {out }}$ is high and can be approximated by one; when INR threshold is in $\left[D_{0}, D_{\max }\right)$, the aggregate interference is approximated by the nearest one very well; when INR threshold exceeds $D_{\max }, P_{\text {out }}$ is very small and drops very fast, so it can be approximated by zero. On the other hand, Gaussian approximation is not suitable for this case. 
B. Case 2: $R_{s}>R\left(D_{0}\right)$, and the aggregate interference is closely approximated by a Gaussian random variable.

When $R_{s} \ll R(D)$, we can still use the corresponding results in [5] and $P_{\text {out }} \approx 1$. Since $R_{s}>R\left(D_{0}\right)$, average number of nodes in $\operatorname{Disk}\left(R_{s}\right)$ is larger than one. When $R(D)$ approaches to $R_{s}$ from the outside $\operatorname{Disk}\left(R_{s}\right)$, average number of nodes in first few rings is not small (it also depends on the path loss exponent), so a typical outage event is when aggregate interference from many nodes in these rings exceeds the threshold $I_{t h}$. When $R(D)$ is less than $R_{s}$, the single INR can not exceed $D_{\max }$, and we need many nodes in a few nearest rings to produce an outage event. The aggregate interference can be approximated by a Gaussian random variable based on the central limit theorem. When the difference of $R_{s}$ and $R\left(D_{0}\right)$ increases, more nodes are involved in the typical outage event, so Gaussian approximation becomes more accurate.

In [6], the authors derived the cumulants of the aggregate interference. In this paper, we introduce a simpler way to obtain the cumulants. The PDF of the interference $I$ coming from a single node (without ordering) is:

$$
f_{I}(I)=\left\{\begin{array}{lc}
\frac{2}{\nu} \frac{I^{-1-2 / \nu}}{R_{\max }^{2}-R_{s}^{2}} & R_{\max }^{-\nu} \leq I \leq R_{s}^{-\nu} \\
0 & \text { otherwise }
\end{array}\right.
$$

The $n^{\text {th }}$ moment of $I$ is

$$
E\left[I^{n}\right]= \begin{cases}\frac{2\left(R_{s}^{2-n \nu}-R_{\max }^{2-n \nu}\right)}{(n \nu-2)\left(R_{\max }^{2}-R_{s}^{2}\right)} & \nu>0 \text { and } \nu \neq \frac{2}{n} \\ \frac{2\left(\ln R_{\max }-\ln R_{s}\right)}{R_{\max }^{2}-R_{s}^{2}} & \nu=\frac{2}{n}\end{cases}
$$

From the system model, $I_{a g}=\sum_{i=1}^{N} I_{i}, N$ is independent of each $I_{i}$ and the mean of $N$ is $\lambda \pi\left(R_{\max }^{2}-R_{s}^{2}\right)$. Poisson point distribution has a property that non-overlapping regions of space are statistically independent. Based on that property, without ordering, the interference from a single node is independent of each other. When $R_{\max }$ goes to infinity, the cumulants of $I_{a g}$ is:

$$
\kappa_{n}=\frac{2 \pi \lambda R_{s}^{2-n \nu}}{n \nu-2}, \nu>2
$$

The results are consistent with eq. (40) in [6].

When $\nu>2$, we can use the first two cumulants to generate Gaussian approximation for $I_{a g}$. The outage probability can be approximated as:

$$
P_{\text {out }}=\operatorname{Pr}\{\gamma>D\} \approx Q\left(\frac{D P_{0}-\frac{2 \lambda \pi R_{s}^{2-\nu}}{\nu-2}}{\sqrt{\frac{\lambda \pi R_{s}^{2-2 \nu}}{\nu-1}}}\right)
$$

where $Q(x)=1 / \sqrt{2 \pi} \int_{x}^{\infty} \exp \left(-u^{2} / 2\right) d u$ is the $\mathrm{Q}$ function. When we use higher order cumulants of $I_{a g}$, more accurate approximations such as Edgeworth expansion or shifted lognormal may be derived [6].

Fig. 3 shows the simulation result for Case 2. From Fig. 3, the aggregate interference is well approximated by Gaussian and Edgeworth expansion. Edgeworth expansion uses the first three cumulants, and is more accurate than the Gaussian one.

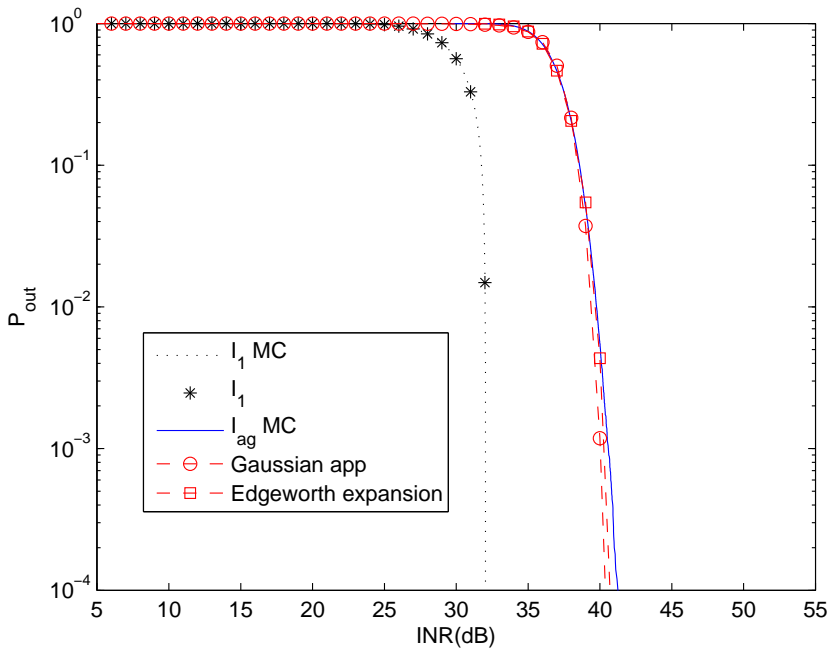

Fig. 3. Outage probability for Case 2. $\nu=4, R_{s}=31.6 \mathrm{~m}, R\left(D_{0}\right)=18 \mathrm{~m}$, $R_{0}=200 \mathrm{~m}, R_{\max }=10^{3} \mathrm{~m}$ and $\lambda=1 \times 10^{-3}\left[\right.$ nodes $\left./ \mathrm{m}^{2}\right]$. The dot line is the simulation result of the nearest interference; the star is theoretical result of the nearest interference; the solid line is the simulation result of the aggregate interference; the dash line with circles is Gaussian approximation; the dash line with squares is Edgeworth expansion Gaussian approximation in [6] (eq. 19).

Since the outage probability drops very fast after the critical value, the difference of two approximations is quite small in terms of INR. Gaussian approximation is a simple and accurate one for Case 2 (but not for Case 1).

C. Case 3: $R_{s}<R\left(D_{0}\right)$ but not $R_{s} \ll R\left(D_{0}\right)$, and neither the nearest node approximation nor Gaussian one is accurate; higher order cumulant approximations or others are required.

When $R_{s}<R\left(D_{0}\right)$, but not $R_{s} \ll R\left(D_{0}\right)$, a typical outage event is when the combination of a few nearest nodes interference exceeds the threshold. The nearest interference approximation is not accurate, and gives us a lower bound for the aggregate one. On the other hand, the number of a few nearest nodes is not large, so Gaussian approximation is not accurate too, but it gives us a critical value to separate the high and low outage range, and a brick wall function using that critical value may be a good approximation for some systems.

\section{REFERENCES}

[1] S. Haykin, "Cognitive radio: brain-empowered wireless communications," IEEE Journal on Selected Areas in Communications, vol.23, no.2, pp. 201-220, Feb. 2005

[2] E.S. Sousa and J.A. Silvester, "Optimum transmission ranges in a directsequence spread-spectrum multihop packet radio network," IEEE Journal on Selected Areas in Communications, vol.8, no.5, pp.762-771, Jun 1990

[3] E.S. Sousa, "Performance of a spread spectrum packet radio network link in a Poisson field of interferers," IEEE Transactions on Information Theory, vol.38, no.6, pp.1743-1754, Nov 1992

[4] J. Ilow and D. Hatzinakos, "Analytic alpha-stable noise modeling in a Poisson field of interferers or scatterers," IEEE Transactions on Signal Processing, vol.46, no.6, pp.1601-1611, Jun 1998

[5] V. Mordachev and S. Loyka, "On node density - outage probability tradeoff in wireless networks," IEEE Journal on Selected Areas in Communications, vol.27, no.7, pp.1120-1131, September 2009

[6] A. Ghasemi and E.S. Sousa, "Interference Aggregation in SpectrumSensing Cognitive Wireless Networks," IEEE Journal of Selected Topics in Signal Processing, vol.2, no.1, pp.41-56, Feb. 2008 